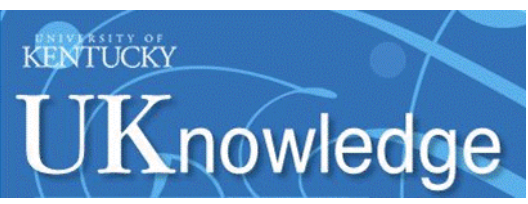

University of Kentucky

UKnowledge

3-14-2012

\title{
Drug Dosage Individualization Based on a Random-Effects Linear Model
}

Francisco J. Diaz

University of Kansas

Myladis R. Cogollo

Universidad EAFIT, Colombia

Edoardo Spina

University of Messina, Italy

Vincenza Santoro

University of Messina, Italy

Diego M. Rendon

Secretaria de Hacienda, Colombia

See next page for additional authors

Follow this and additional works at: https://uknowledge.uky.edu/psychiatry_facpub

Part of the Psychiatry and Psychology Commons

Right click to open a feedback form in a new tab to let us know how this document benefits you.

\section{Repository Citation}

Diaz, Francisco J.; Cogollo, Myladis R.; Spina, Edoardo; Santoro, Vincenza; Rendon, Diego M.; and de Leon, Jose, "Drug Dosage Individualization Based on a Random-Effects Linear Model" (2012). Psychiatry Faculty Publications. 12.

https://uknowledge.uky.edu/psychiatry_facpub/12

This Article is brought to you for free and open access by the Psychiatry at UKnowledge. It has been accepted for inclusion in Psychiatry Faculty Publications by an authorized administrator of UKnowledge. For more information, please contact UKnowledge@lsv.uky.edu. 


\section{Drug Dosage Individualization Based on a Random-Effects Linear Model}

Digital Object Identifier (DOI)

http://dx.doi.org/10.1080/10543406.2010.547264

\section{Notes/Citation Information}

Published in Journal of Biopharmaceutical Statistics, v. 22, issue 3, p. 463-484.

This is an Accepted Manuscript of an article published by Taylor \& Francis in Journal of

Biopharmaceutical Statistics on 03/14/2012, available online: http://wwww.tandfonline.com/ 10.1080/ 10543406.2010.547264

\section{Authors}

Francisco J. Diaz, Myladis R. Cogollo, Edoardo Spina, Vincenza Santoro, Diego M. Rendon, and Jose de Leon 


\title{
Drug Dosage Individualization Based on a Random-Effects Linear Model
}

\author{
Francisco J. Diaz, ${ }^{1}$ Myladis R. Cogollo, ${ }^{2}$ Edoardo Spina, ${ }^{3}$ \\ Vincenza Santoro, ${ }^{3}$ Diego M. Rendon, ${ }^{4}$ Jose de Leon ${ }^{5}$
}

${ }^{1}$ Corresponding author. Department of Biostatistics, The University of Kansas Medical Center, Mail Stop 1026, 3901 Rainbow Blvd., Kansas City, KS 66160, USA. Phone: (913) 945-7006. Email: fdiaz@kumc.edu.

2 Department of Basic Sciences, Universidad EAFIT, A.A. 3300, Medellin, Colombia.

3 Section of Pharmacology, Department of Clinical and Experimental Medicine and Pharmacology, University of Messina and IRCCS Centro Neurolesi "Bonino-Pulejo", Policlinico Universitario di Messina, Via Consolare Valeria, 98125, Messina, Italy.

${ }^{4}$ Financial Analysis Unit. Secretaria de Hacienda, Medellin, Colombia.

${ }^{5}$ Mental Health Research Center, Eastern State Hospital, 627 West Fourth St., Lexington, KY, 40508, USA.

KEY WORDS: Bayesian feedback; Bayes theorem; clozapine; decision theory; drug dosage individualization; random effects linear models; personalized medicine; population pharmacokinetics; therapeutic drug monitoring; therapeutic window.

SHORT TITLE: Drug Dosage Individualization and Linear Mixed Models. 


\begin{abstract}
This article investigates drug dosage individualization when the patient population can be described with a random effects linear model of a continuous pharmacokinetic or pharmacodynamic response. Specifically, we show through both decision-theoretic arguments and simulations that a published clinical algorithm may produce better individualized dosages than some traditional methods of therapeutic drug monitoring. Since empirical evidence suggests that the linear model may adequately describe drugs and patient populations, and linear models are easier to handle than the nonlinear models traditionally used in population pharmacokinetics, our results highlight the potential applicability of linear mixed models to dosage computations and personalized medicine.
\end{abstract}




\section{Introduction}

In the pharmacological treatment of some chronic illnesses, the clinician must search for an appropriate drug dosage $D$ that, after being administered during a pre-specified time period to a particular patient, will maximize the probability that a continuous pharmacokinetic or pharmacodynamic response $Y_{D}$ takes a value between two pre-specified values $l_{1}$ and $l_{2}$. Examples of these treatments are those based on drugs having a narrow therapeutic range, which is the range of plasma (or blood) drug concentrations that yield the desired pharmacological effect without toxicity; in this case, a pharmacokinetic response of interest $\left(Y_{D}\right)$ is usually the steady-state trough plasma drug concentration in the patient, which is measured during routine therapeutic drug monitoring (TDM) (Shirrell et al., 1999). For instance, when administering the antipsychotic drug clozapine to a patient with schizophrenia, the clinician must make efforts to avoid plasma clozapine concentrations in the patient reaching very low or high values, since low concentrations will not reduce psychotic symptoms and high concentrations will increase the risk of severe sedation, seizures and other undesirable effects (Sabaawi et al., 2006). In this example, $D$ is clozapine dosage and $Y_{D}$ is plasma clozapine concentration.

Another (classic) example in which the plasma (or blood) concentration of the treatment drug is a response of interest may be found during the treatment for heroin addiction based on methadone. An effective methadone-maintenance treatment requires that the minimum methadone concentration in the patient's blood be kept above the threshold of heroin-withdrawal symptoms, and that the maximum concentration be kept below the threshold of the appearance of narcotic effects (Dole, 1980). Whereas low methadone concentrations do not overcome heroin-withdrawal symptoms and also increase the risk of relapsing into heroin use, high concentrations will sedate the patient, preventing him/her from enjoying a normal and fruitful life. In this example, $D$ is methadone dosage and $Y_{D}$ is blood methadone concentration. 
There are pharmacological treatments in which the clinician wants to control a continuous response other than plasma concentration of the treatment drug. An example is the use of the anti-coagulant drug warfarin to prevent blood clotting in patients with risk of thrombotic episodes. To monitor the treatment and adjust warfarin dosage, regular blood tests must be performed to measure the clotting tendency of the patient's blood; this measure is usually given as an international normalized ratio, INR (Cuadrado et al., 2000). The objective of dosage adjustment is to find an optimum dosage that produces an anti-coagulation intensity between minimum and maximum pre-specified values. Lower dosages than this minimum may not be effective (putting the patient at risk of a new thrombotic episode), and higher dosages than the maximum may produce serious hemorrhages. Here, $D$ is warfarin dosage and $Y_{D}$ is the INR, which is a pharmacodynamic response of interest.

In the above examples and those similar, the clinician usually assumes that the patient's continuous response (drug plasma or blood concentration in the first 2 examples and INR in the third example) will become stable after administering a constant drug dosage for a known, fixed period of time. Further, the clinician must take steps to find a dosage that, after being administered for that period of time, maximizes the probability that the patient's response reaches a value that is not too low or too high, and therefore maximizes the chance of relieving the illness symptoms and minimizes the risk of experiencing undesirable or toxic effects. Finding this dosage is usually difficult, since there is usually wide variability across individual patients in response to the same pharmacological treatment. This response variability may be due to personal (e.g., age or gender), genetic, or environmental (e.g., drug-drug interactions) influences on pharmacokinetic and/or pharmacodynamic parameters, or even to patient compliance (Nies, 2001). Efforts have been made to develop drug-dosage individualization procedures based on statistical models that take into account this variability (for a review, 
see Diaz et al., 2007). Among these procedures, those based on mixed effects models seem to be very promising.

The application of mixed effects models to the individualization of drug dosages was pioneered by Sheiner and his collaborators (see Vozeh et al., 1981; Sheiner and Beal, 1982). Underlying this application is the basic idea that a random coefficient in a mixed effects model can be viewed as a parameter that is a characteristic constant for a particular patient in the population of patients, but that varies across patients (see Sheiner and Beal, 1980; Whiting et al., 1986). According to this idea, the variability of a random coefficient reflects a real variation in the biological and environmental variables that shape each person in the population as an individual; variability is not a mere mathematical artifact for dealing with a population's heterogeneity. Thus, a mixed effects model includes parameters with constant values representing the whole population (fixed effects) and parameters with varying values whose particular values represent particular patients (random effects). Usually, these parameters measure or reflect patients' pharmacokinetic or pharmacodynamic characteristics. For a history of both the theory and applications of mixed effects models in pharmacology, see Pillai et al., 2005.

In practice, any drug dosage individualization procedure based on a population mixed effects model utilizes Bayes' theorem. Before applying a procedure to a particular patient, the fixed effects and variance-covariance matrices of the random effects and errors must be estimated by using one or more samples of patients, or using previous pharmacological knowledge. Then, the estimated distribution of the random coefficients is conceived of as a prior distribution for these coefficients, and a few response measurements (e.g., drug plasma concentrations) from the patient are used to compute the conditional distribution of the random coefficients given those measurements. A summary measure of this conditional distribution, such as its mean or mode vector, is 
considered a predictor of the true values of the patient's coefficients, and therefore used to compute the patient's individualized dosage. According to this methodology, which is usually called "Bayesian feedback", a prior distribution models real-world random variables (i.e., models the variability of some pharmacokinetic or pharmacodynamic parameters in the patient population). Thus, the prior distribution is not interpreted as a representation of the clinician's system of subjective beliefs about hypothetical, constant population parameters, which would be an orthodox, classic interpretation of the prior distribution underlying Bayes' theorem. Failing to recognize that statistical practice may profit from these two different philosophical conceptions of prior distributions, rather than from the latter one alone, may have contributed to a misunderstanding of the Bayesian feedback methodology in the statistical community, which struggled for decades to understand the philosophical implications of Bayes' theorem.

Diaz et al. (2007) considered the individualization of a drug dosage when the natural log of steady-state drug plasma concentration-to-dosage ratio can be described using a random intercept linear model. In their model, it is assumed that no covariate has a random effect. Specifically, the studied model was

$$
\log \left(\frac{Y_{D}}{D}\right)=\alpha+\boldsymbol{\beta}^{T} \boldsymbol{X}+\epsilon
$$

where $Y_{D}$ is a trough steady-state drug plasma concentration, $D$ is the steady-state dosage, $\boldsymbol{X}$ is a vector with $r$ covariates, $\boldsymbol{\beta}$ is a vector with regression coefficients that are population constants, and $\alpha$ is a characteristic constant of each patient. At the population level, it is assumed that $\alpha$ is a $N\left(\mu_{\alpha}, \sigma_{\alpha}^{2}\right)$ random variable, and that $\epsilon$ is an intra-individual $N\left(0, \sigma_{\epsilon}^{2}\right)$ random error. Moreover, it is assumed that $\alpha$ is independent of $\epsilon$. Diaz et al. (2007) suggested interpreting the quantity $\gamma=\left(\alpha-\mu_{\alpha}\right) / \sigma_{\alpha}$ as a standardized, covariate-adjusted index of metabolic activity, and suggest a procedure for empirically validating this index. (The faster a patient's body eliminates the drug, 
the lower his/her value of $\gamma$.) This interpretation is a direct consequence of the idea that random coefficients may be viewed as constants for a particular patient. In an independent study, Hu and Zhou (2008) suggested a more precise interpretation of $\alpha$ (and therefore of $\gamma$ ) and a rationale for using model (1) in dosage computations (see also Hu et al., 2009). Assuming linear pharmacokinetics, if $C L / F$ is the patient's apparent clearance, the quantity $e^{\alpha+\boldsymbol{\beta}^{T} \boldsymbol{X}}$ is a multiple of $(C L / F)^{-1}$. Thus, $e^{\alpha}$ may be viewed as the portion of $C L / F$ that is not explained by the covariates in $\boldsymbol{X}$. Therefore, $\gamma$ is a covariate-adjusted proxy for apparent clearance; and model (1) quantifies the effects of covariates on apparent clearance (Hu and Zhou, 2008), which is the most important pharmacokinetic quantity to consider when designing a dosage regimen for long-term drug administration.

Diaz et al. (2007) proposed a clinical algorithm for drug-dosage individualization based on model (1). The algorithm was deduced from decision-theoretic concepts and consists of a series of steps that the clinician should follow in order to find an appropriate dosage for a particular patient, assuming that model (1) adequately represents the patient population. Before applying the algorithm to a particular patient, the population parameters $\boldsymbol{\beta}, \mu_{\alpha}, \sigma_{\alpha}^{2}$ and $\sigma_{\epsilon}^{2}$ have to be estimated using a sample of patients. A simulation study using a clozapine model built with a US patient sample suggested that the algorithm will perform reasonably well, even including the possibility that parameter estimates are not very close to the true parameter values, and even if clozapine dosages are rounded to the closest multiple of the smallest dosage available from clozapine manufacturers, $25 \mathrm{mg} /$ day (2007).

A difficult problem when individualizing a drug dosage using a population mixed effects model is to decide how many drug plasma concentrations to obtain from the patient, such that an optimum dosage for the patient is computed. Solutions to this problem usually are ad hoc or lack clear justifications. However, Diaz et al. (2007) 
provided a theoretical framework that enables a solution to this problem for model (1). They introduced the concept of $\omega$-optimum dosage for a patient with metabolic index $\gamma$, which, for $\omega$ close to 1 , is essentially a dosage $D$ that nearly maximizes the conditional probability $P\left(l_{1}<Y_{D}<l_{2} \mid \gamma\right)$, where $\left(l_{1}, l_{2}\right)$ is the range of desired plasma concentrations. Thus, the optimum number of plasma concentrations that must be obtained from the patient is the one that allows computing an $\omega$-optimum dosage for a pre-specified, high proportion of patients in the population.

When discussing Diaz et al.'s article (2007), it is important to differentiate between model (1) and their proposed algorithm for drug dosage individualization. Whereas the former is a description of the patient population, the latter is a series of steps for finding an optimum dosage for a particular patient. The algorithm's applicability relies on the assumption that model (1) correctly describes the population. Diaz et al. (2007) found that model (1) was useful in studying the relationship between plasma concentrations of the antipsychotic clozapine and the covariates gender and smoking, controlling for clozapine dosage. In addition, Diaz et al. (2008) and Botts et al. (2008) suggest that a slightly more general version of model (1) may be useful for investigating drug-drug interactions. In particular, the model allowed the finding that smoking modifies the size of the effect of the anticonvulsant valproic acid on plasma clozapine concentrations (Diaz et al., 2008), and that it also modifies the size of the effect of the anticonvulsant lamotrigine on the plasma concentrations of the antipsychotic olanzapine (Botts et al., 2008). Hu and Zhou (2008) described an even more general version of model (1) that included transformed covariates and both peak and trough steady-state plasma concentrations. They proposed using their linear model to investigate the sensitivity of results from population pharmacokinetics analyses, and studied 3 drugs (2 biologicals and 1 small molecule) using large, multinational patient samples. However, although the objectives of Hu and Zhou's study did not include searching for empirical evidence 
in favor of the linear model, they found a remarkable agreement between the conclusions obtained by using this model and those obtained by using traditional, pharmacokinetic non-linear models. In particular, the average covariate-based dosing adjustment factors provided by linear and non-linear models were essentially the same regardless of the investigated drug or covariate. The 4 above studies (Diaz et al., 2007, 2008; Botts et al., 2008; and $\mathrm{Hu}$ and Zhou, 2008), which used a total of 6 different samples of patients representing people from several countries and 5 different drugs, reported evidence of relatively good fits of the random-intercept linear models to the data, although some caution was advised when there was a possibility of comedication-time interactions $(\mathrm{Hu}$ and Zhou, 2008; Botts et al., 2008).

Whereas there is empirical evidence supporting model (1) and some of its generalizations, at least for some drugs, Diaz et al.'s algorithm has been tested only through simulations (Diaz et al., 2007). However, the simulation results, the above theoretical developments and evidence in favor of model (1) or similar models, and other authors' successful application of Bayesian feedback, suggest that the algorithm is a potential tool for clinical practice and TDM that deserves a closer investigation. In particular, collaborative effort is necessary for precisely estimating population parameters using large samples of patients from particular populations and for particular drugs, and it is essential to compare the performance of the algorithm with that of currently accepted methods that are regularly used in TDM.

Model (1) does not include covariates with random effects, i.e., covariates whose corresponding regression coefficients vary across patients. In model (1), the heterogeneity of the individuals is determined only by differences in the intercepts (the $\alpha$ 's). This is a limitation, because the model does not allow for the possibility of covariates whose effects on $Y_{D}$ vary among patients who have the same values on the other covariates and are under the same dosage. In fact, additional analyses of the clozapine data in Diaz 
et al. (2008), which are described in Section 4 below, indicated that the goodness of the fit of the random-intercept linear model described in these authors' article may be improved by incorporating random effects for some covariates, including smoking. In particular, this suggests that smoking has an effect size on plasma clozapine concentrations that varies from patient to patient and, perhaps more importantly, that the extent to which smoking modifies the effect of valproic acid on plasma clozapine concentrations may vary from patient to patient. We do not know whether this differential smoking effect is due to differences in smoking behavior, to differences in personal, genetic or environmental characteristics of the patients, or to all of these factors (although this difference may not be due to the number of cigarettes smoked daily; Diaz et al., 2008). However, the possible existence of covariates with random effects affecting a continuous pharmacokinetic response has motivated us to investigate whether the drug-dosage individualization algorithm in Diaz et al. (2007) is applicable to the situation where the patient belongs to a population that can be described by a linear model incorporating covariates with random effects.

This article investigates the clinical algorithm proposed by Diaz et al. (2007), and the use of linear mixed models in drug dosage individualization. Specifically, this article has three objectives: 1) to describe how Diaz et al.'s algorithm can be applied to situations where some clinical and demographic covariates have random effects on a continuous pharmacokinetic or pharmacodynamic response $Y_{D}$, and to situations that include the linear models fitted in $\mathrm{Hu}$ and Zhou (2008), Diaz et al. (2008) and Botts et al. (2008) (in these 3 studies, the $D$ in model (1) was replaced by $D^{d}$, where $d$ was a parameter that was estimated); 2) to report decision-theoretic arguments that show that the above algorithm may produce better dosages than another method which has traditionally been used in TDM; and 3) to compare the algorithm with this other method using simulated populations of patients taking clozapine. With this article, we 
also want to highlight the potentially wide applicability of linear mixed models to drug dosage computations and personalized medicine.

Although at present most of the applications of mixed effects models to drugdosage individualization have been restricted to plasma drug concentrations as the response variable, other types of response are possible. Thus, in this article, we will be concerned with continuous pharmacokinetic or pharmacodynamic responses that can be assumed to become stable after an administration of a constant drug dosage for a known fixed period of time. Hereafter, in model (1) and all models investigated in this article, $Y_{D}$ will represent the value of a (positive) continuous pharmacokinetic or pharmacodynamic response measured after administering a constant drug dosage $D$ during such time period. Of course, this time period depends on the particular drug and response. For instance, when the response is plasma (or blood) drug concentration, this time period usually corresponds to at least 5 drug half-lives, and the drug concentrations measured after that period are usually termed "steady-state" (Winter, 2004). However, a continuous response may not necessarily be a drug plasma concentration, but may be an index of efficacy or toxicity of the drug, a measure of clinical benefit or harm, a clinical endpoint, or even a continuous surrogate marker (Molenberghs et al., 2008). The basic goal of a drug-dosage individualization process is to determine an optimum dosage $D$ for a particular patient, which allows him/her to reach a response $Y_{D}$ that lies within a range of desired responses $\left(l_{1}, l_{2}\right)$. (The interval $\left(l_{1}, l_{2}\right)$ will be called the target response range.)

In Section 2, we describe how Diaz et al.'s algorithm can be used in situations where some covariates have random effects and/or the log of dosage has a fixed regression coefficient. In Section 3, the algorithm is compared with a popular TDM method through theoretical arguments. In Section 4, a model of plasma clozapine concentrations that has covariates with random effects is described, and some computations that 
are necessary for performing Diaz et al.'s algorithm are illustrated. In Section 5, simulations are used to compare the algorithm with the TDM method, assuming accurate parameter estimates. The effects of estimation errors on the algorithm's performance are investigated through simulations in Section 6. Section 7 discusses empirical evidence supporting the investigated linear model, and describes additional advantages of using linear over non-linear models in drug dosage computations. Conclusions are in Section 8.

\section{Applicability of Diaz et al.'s algorithm in a more general setting}

This study considers the model

$$
\log \left(Y_{D}\right)=\psi+\boldsymbol{\eta}^{T} \boldsymbol{Z}+\boldsymbol{\beta}^{T} \boldsymbol{X}+d \log (D)+\epsilon
$$

where $\epsilon$ is defined as in model (1), $\boldsymbol{Z}$ and $\boldsymbol{X}$ are vectors with $k$ and $r$ (possibly transformed) clinical or demographic covariates, respectively, $\boldsymbol{\beta}$ is a vector of regression coefficients that are population constants, and $d$ is a population constant. Here, $\psi$ and $\boldsymbol{\eta}$ are characteristic constants of a particular patient; they vary from patient to patient. At the population level, it is assumed that $\psi$ is a $N\left(\mu_{\psi}, \sigma_{\psi}^{2}\right)$ random variable, that $\boldsymbol{\eta}$ is a $k$-dimensional $N_{k}\left(\boldsymbol{\mu}_{\eta}, \boldsymbol{V}_{\eta}\right)$ random vector, that $(\psi, \boldsymbol{\eta})$ has a joint normal distribution, and that $(\psi, \boldsymbol{\eta})$ and $\epsilon$ are independent from each other. Please note that whereas $\boldsymbol{X}$ includes only covariates with fixed effects (the elements of $\boldsymbol{\beta}$ ), $\boldsymbol{Z}$ includes covariates that have fixed effects (the elements of $\boldsymbol{\mu}_{\eta}$ ) and random effects (the elements of $\boldsymbol{\eta}-\boldsymbol{\mu}_{\eta}$ ). Therefore, in order for $\boldsymbol{\beta}$ and $\boldsymbol{\mu}_{\eta}$ to be identifiable, we assume that none of the covariates in $\boldsymbol{X}$ are in $\boldsymbol{Z}$, and vice versa. In the traditional terminology of mixed linear models, the vectors $\left(\mu_{\psi}, \boldsymbol{\mu}_{\eta}^{T}, \boldsymbol{\beta}^{T}, d\right)^{T}$ and $\left(\psi-\mu_{\psi},\left(\boldsymbol{\eta}-\boldsymbol{\mu}_{\eta}\right)^{T}\right)^{T}$ are called fixed and random effects, respectively (Verbeke and Molenberghs, 2000), with the understanding that $\mu_{\psi}$ and $\psi-\mu_{\psi}$ do not measure the "effects" of any real-world covariate because $\psi$ represents only an intercept. If $Y_{D}$ is drug plasma concentration and linear pharmacokinetics is assumed, $d$ may be fixed at 1 (Hu and Zhou, 2008; Hu et al., 2009). Alternatively, the 
maximum likelihood estimator of $d$ may be used to test the null hypothesis of linear pharmacokinetics, $d=1$; and $d$ should be estimated if there is evidence of nonlinear pharmacokinetics. When $d=1$ and $\boldsymbol{\eta} \equiv 0$, model (2) is essentially model (1); in this sense, model (2) is a generalization of model (1).

For a fixed value of $\boldsymbol{Z}$, if we denote $\alpha=\psi+\boldsymbol{\eta}^{T} \boldsymbol{Z}$ then model (2) can be rewritten as

$$
\log \left(\frac{Y_{D}}{D^{d}}\right)=\alpha+\boldsymbol{\beta}^{T} \boldsymbol{X}+\epsilon
$$

with $\alpha \sim N\left(\mu_{\alpha}(\boldsymbol{Z}), \sigma_{\alpha}^{2}(\boldsymbol{Z})\right)$, where $\mu_{\alpha}(\boldsymbol{Z})=\mu_{\psi}+\boldsymbol{\mu}_{\eta}^{T} \boldsymbol{Z}$ and $\sigma_{\alpha}^{2}(\boldsymbol{Z})=\operatorname{Var}\left(\psi+\boldsymbol{\eta}^{T} \boldsymbol{Z}\right)$. Thus, for fixed $\boldsymbol{Z}$, model (3) is analogous to model (1), although in model (3) the parameters of $\alpha$ depend on $\boldsymbol{Z}$ and the dosage $D$ has been "transformed" to $D^{d}$. Now assume that model (2) describes adequately a population of patients, and that a clinician wants to find an appropriate, individualized dosage $D$ for a patient who belongs to this population and has a constant but unknown $\alpha$. The above analogy and the fact that $\boldsymbol{Z}$ is known and fixed throughout the entire individualization process, suggest that Diaz et al.'s algorithm can be implemented in the following way in the context of model (2). Here, it is assumed that the values of $\mu_{\alpha}(\boldsymbol{Z}), \sigma_{\alpha}^{2}(\boldsymbol{Z}), \boldsymbol{\beta}, \sigma_{\epsilon}^{2}$ and $d$ are known (or have been estimated with acceptable precision before applying the algorithm), which implies that the distribution $N\left(\mu_{\alpha}(\boldsymbol{Z}), \sigma_{\alpha}^{2}(\boldsymbol{Z})\right)$ is our prior distribution of $\alpha$. When model (2) holds, the steps of the algorithm are as follows:

Step 1: By using $\widehat{\alpha}_{1}^{\prime}=\mu_{\alpha}(\boldsymbol{Z})$ and $C_{0}^{*}=\sqrt{l_{1} l_{2}}$, compute the initial dosage

$$
D_{1}=\left(C_{0}^{*} e^{-\widehat{\alpha}_{1}^{\prime}-\boldsymbol{\beta}^{T} \boldsymbol{X}}\right)^{1 / d} .
$$

Next, administer $D_{1}$ to the patient during an appropriate time period so that the response becomes stable, and then measure the stabilized response $Y_{D_{1}}$.

Step $i, i \geq 2$ : By using the dosage-response pairs $\left(D_{j}, Y_{D_{j}}\right), j=1,2, \ldots, i-1$, which were obtained in the $i-1$ previous steps, compute the $i$-th dosage 


$$
D_{i}=\left(C_{0}^{*} e^{-\widehat{\alpha}_{i}^{\prime}-\boldsymbol{\beta}^{T} \boldsymbol{X}}\right)^{1 / d}
$$

where $\widehat{\alpha}_{i}^{\prime}$ is a predictor of $\alpha$ given by

$$
\widehat{\alpha}_{i}^{\prime}=\left(1-\lambda_{i} \sqrt{\rho^{-1}-1}\right)\left(\frac{1}{i-1} \sum_{j=1}^{i-1} \log \left(\frac{Y_{D_{j}}}{D_{j}^{d}}\right)-\boldsymbol{\beta}^{T} \boldsymbol{X}\right)+\lambda_{i} \sqrt{\rho^{-1}-1} \mu_{\alpha}(\boldsymbol{Z}),
$$

with

$$
\rho=\rho(\boldsymbol{Z})=\sigma_{\alpha}^{2}(\boldsymbol{Z}) /\left\{\sigma_{\alpha}^{2}(\boldsymbol{Z})+\sigma_{\epsilon}^{2}\right\}
$$

and $\lambda_{i}, i \geq 1$, defined by Eq. (12) in Diaz et al. (2007). (We stress that, in the context of model (2), $\rho$ depends on $\boldsymbol{Z}$.) Administer the new dosage $D_{i}$ to the patient until the response stabilizes, and then measure the produced, stabilized response $Y_{D_{i}}$. Since we are assuming that model (2) holds, we have that $Y_{D_{i}}=D_{i}^{d} e^{\alpha+\boldsymbol{\beta}^{T} \boldsymbol{X}+\epsilon_{i}}, i \geq 1$, where $\alpha=\psi+\boldsymbol{\eta}^{T} \boldsymbol{Z}$ is a constant number for the patient and the $\epsilon_{i}$ 's are $N\left(0, \sigma_{\epsilon}^{2}\right)$. The $\epsilon_{i}$ 's are assumed to be mutually independent.

For $i \geq 2, \widehat{\alpha}_{i}^{\prime}$ is the mean of the conditional distribution of $\alpha$ given $\log \left(Y_{D_{j}} / D_{j}^{d}\right)-$ $\boldsymbol{\beta}^{T} \boldsymbol{X}, j=1, \ldots, i-1$. By using Eq. (3) and a decision-theoretic argument analogous to the proof of Theorem 3.1 in Diaz et al. (2007), it can be shown that, when model (2) holds, Diaz et al.'s algorithm is optimal in the sense that, at the $i$-th algorithm step, $\widehat{\alpha}_{i}^{\prime}$ and $C_{0}^{*}$ are the predictor $\widehat{\alpha}_{i}$ of $\alpha$ and the target median patient's response $C_{0}$, respectively, that minimize the Bayes risk function

$$
R\left(\widehat{\alpha}_{i}, C_{0}\right)=1-P\left(l_{1}<Y_{D_{i}}<l_{2}\right), \quad\left(\widehat{\alpha}_{i}, C_{0}\right) \in \mathcal{G}_{i} \times(0, \infty),
$$

where $\mathcal{G}_{i}$ is the set of all predictors $\widehat{\alpha}_{i}$ of $\alpha$ that are normally distributed, satisfy $E\left[\widehat{\alpha}_{i}\right]=$ $\mu_{\alpha}(\boldsymbol{Z})$ and are independent of $\epsilon_{i}$, and it is assumed that the interval $(0, \infty)$ includes any possible response. (Please keep in mind that the value of $\boldsymbol{Z}$ is fixed during the whole dosage individualization process, since the algorithm is applied to a particular patient.) Thus, essentially, Diaz et al.'s algorithm aims at finding a good predictor of $\alpha$ in the sense that this predictor maximizes the clinician's degree of confidence that the response $Y_{D}$ will fall within the target range $\left(l_{1}, l_{2}\right)$. 
Suppose that a population of patients satisfy model (2), and that a particular patient from the population has characteristic constants $\psi$ and $\boldsymbol{\eta}$ and, therefore, a characteristic $\alpha=\psi+\boldsymbol{\eta}^{T} \boldsymbol{Z}$. Assume that Diaz et al.'s algorithm is applied to this patient. An important question is: How many algorithm steps are necessary to obtain an appropriate dosage for the patient? This question is answered by using the concept of $\omega$-optimum dosage (Diaz et al., 2007). Specifically, in the context of model (2), for $0<\omega<1$, an $\omega$-optimum dosage for the patient is a dosage $D$ that satisfies

$$
P\left(l_{1}<Y_{D}<l_{2} \mid \gamma\right) \geq \omega\left\{\sup _{i \geq 1} P\left(l_{1}<Y_{D_{i}}<l_{2} \mid \gamma\right)\right\}
$$

where $\gamma=\left[\alpha-\mu_{\alpha}(\boldsymbol{Z})\right] / \sigma_{\alpha}(\boldsymbol{Z})$. Thus, when model (2) holds, the largest attainable probability that the response of the patient reaches a value within the target range $\left(l_{1}, l_{2}\right)$ is $m$, and the minimum number of algorithm steps that are necessary to reach an $\omega$-optimum dosage for the patient is $I(\gamma, \omega)$, where $m$ and $I(\gamma, \omega)$ are defined by Eqs. (14) and (16) in Diaz et al. (2007), respectively. (Please observe that, besides depending on $\gamma$ and $\omega, I(\gamma, \omega)$ depends on both $m$ and $\rho$, where $\rho$ is given by Eq. (7) if model (2) holds.)

In practice, since the patient's $\gamma$ is unknown, it is necessary to stop the algorithm at the earliest step that yields an $\omega$-optimum dosage for at least a fraction $p$ of patients, $0<p<1$. When model (2) holds, the minimum number of algorithm steps that are necessary to obtain an $\omega$-optimum dosage for at least $p \times 100 \%$ of the patients in the population is $i^{*}$, which is defined by Eq. (18) in Diaz et al. (2007). In practice, the values of $\omega$ and $p$ should be fixed close to 1 before starting the algorithm. [The above statements can be proven analogously to the proof of Theorem 3.2 in Diaz et al., 2007.] Please note that, in the context of model $(2), i^{*}$ depends on the particular value that $\boldsymbol{Z}$ takes on in the patient, although $i^{*}$ does not depend on $\boldsymbol{X}$. Moreover, although the definition of an $\omega$-optimum dosage given by (9) formally depends on an infinite sequence of future dosages, only a finite number of administered dosages is needed to 
compute a dosage that has at least a $p \times 100 \%$ possibility of being $\omega$-optimum for the patient. This number is $i^{*}-1$.

\section{A comparison with therapeutic drug monitoring}

Diaz et al.'s algorithm may be considered an optimal method for dosage adjustment in a TDM setting when the patient belongs to a population that satisfies model (2) and $Y_{D}$ is drug plasma concentration. To understand why this may be so, let us compare Diaz et al.'s algorithm with a popular method for dosage adjustment which is advocated in a number of pharmacology textbooks. This method, which assumes linear pharmacokinetics, adjusts the patient's dosage using the formula

$$
\text { Adjusted dosage }=\frac{\text { Previous dosage }}{\text { Measured concentration }} C_{0},
$$

where $C_{0}$ is a target drug steady-state trough concentration. Let $A$ and $B$ be two patients from a population that satisfies model (2) with $d=1$, who are independently treated with the drug. [The condition $d=1$ insures linear pharmacokinetics $(\mathrm{Hu}$ and Zhou, 2008; Hu et al., 2009).] Assume that the two patients have the same covariate values in $\boldsymbol{X}$ and $\boldsymbol{Z}$, and have the same but unknown value of $\alpha$, where $\alpha=\psi+\boldsymbol{\eta}^{T} \boldsymbol{Z}$, so that the patients are closely comparable. Suppose that we want to obtain steady-state trough plasma concentrations that fall within a target range $\left(l_{1}, l_{2}\right)$, and that patients $A$ and $B$ are initially administered dosages $D_{1}$ and $D_{b, 1}$, respectively, where $D_{1}=D_{b, 1}$ and $D_{1}$ is given by Eq. (4). Suppose that the dosage for patient $A$ is adjusted $n$ times using Diaz et al.'s algorithm as described in Section 2 (using $C_{0}^{*}$ as target concentration and $\widehat{\alpha}_{i}^{\prime}$ as a predictor of $\alpha$ ), and that the dosage for patient $B$ is adjusted $n$ times using (10), where $C_{0}$ may not be equal to $C_{0}^{*}$. The following argument shows that, after any dosage adjustment, patient $A$ will have a higher chance of reaching the target concentration range than patient $B$.

Let $\left(D_{i}, Y_{D_{i}}\right), i=1, \ldots, n+1$, be the dosage-concentration pairs obtained from 
patient $A$. Thus, $Y_{D_{i}}=D_{i} e^{\alpha+\boldsymbol{\beta}^{T} \boldsymbol{X}+\epsilon_{i}}, i=1, \ldots, n+1$, where the $\epsilon_{i}$ 's are mutually independent random errors with a $N\left(0, \sigma_{\epsilon}^{2}\right)$ distribution. From patient $B$, we obtain dosage-concentration pairs $\left(D_{b, i}, Y_{D_{b, i}}\right), i=1, \ldots, n+1$, which satisfy $D_{b, i}=\left(D_{b, i-1} / Y_{D_{b, i-1}}\right) C_{0}$ and $Y_{D_{b, i}}=D_{b, i} e^{\alpha+\boldsymbol{\beta}^{T} \boldsymbol{X}+\epsilon_{b, i}}$, where the $\epsilon_{b, i}$ 's are $N\left(0, \sigma_{\epsilon}^{2}\right)$ random errors that may be reasonably assumed to be mutually independent and independent of the $\epsilon_{i}$ 's. But, for $i \geq 2$,

$$
Y_{D_{b, i}}=\left(C_{0} e^{-\widehat{\alpha}_{i}-\boldsymbol{\beta}^{T} \boldsymbol{X}}\right)\left(e^{\alpha+\boldsymbol{\beta}^{T} \boldsymbol{X}+\epsilon_{b, i}}\right)
$$

where $\widehat{\alpha}_{i}=\log \left(Y_{D_{b, i-1}} / D_{b, i-1}\right)-\boldsymbol{\beta}^{T} \boldsymbol{X}$. Thus, applying formula (10) is equivalent to applying Diaz et al.'s algorithm, except that $C_{0}$ and $\widehat{\alpha}_{i}$ are used in place of $C_{0}^{*}$ and $\widehat{\alpha}_{i}^{\prime}$ [cf. Eq. (5) with $d=1$ ]. Now observe that $\widehat{\alpha}_{i} \in \mathcal{G}_{i}$, where $\mathcal{G}_{i}$ is defined after Eq. (8). Thus, if $C_{0} \neq C_{0}^{*}$, then

$$
P\left(l_{1}<Y_{D_{b, i}}<l_{2}\right)<P\left(l_{1}<Y_{D_{i}}<l_{2}\right),
$$

for all $i=1, \ldots, n+1$ (see proof of Theorem 3.1 in Diaz et al., 2007.) To see why inequality (12) is also valid when $C_{0}=C_{0}^{*}$, denote $\widehat{\alpha}_{i}^{\prime \prime}=\frac{1}{i-1} \sum_{j=1}^{i-1}\left(\log \left(\frac{Y_{D_{j}}}{D_{j}}\right)-\boldsymbol{\beta}^{T} \boldsymbol{X}\right)$. Note that $E\left[\widehat{\alpha}_{i} \mid \alpha\right]=\alpha=E\left[\widehat{\alpha}_{i}^{\prime \prime} \mid \alpha\right]$. For $i \geq 2, E\left[\left(\widehat{\alpha}_{i}-\alpha\right)^{2} \mid \alpha\right]=\sigma_{\epsilon}^{2} \geq \sigma_{\epsilon}^{2} /(i-1)=$ $E\left[\left(\widehat{\alpha}_{i}^{\prime \prime}-\alpha\right)^{2} \mid \alpha\right]$. Thus, if $C_{0}=C_{0}^{*}$ and $i \geq 2, E\left[\left(\widehat{\alpha}_{i}-\alpha\right)^{2}\right] \geq E\left[\left(\widehat{\alpha}_{i}^{\prime \prime}-\alpha\right)^{2}\right]>$ $E\left[\left(\widehat{\alpha}_{i}^{\prime}-\alpha\right)^{2}\right]$ because $\widehat{\alpha}_{i}^{\prime}$ is a Bayes estimator of $\alpha$ under the squared error loss but $\widehat{\alpha}_{i}^{\prime \prime}$ is not; since $\widehat{\alpha}_{i} \in \mathcal{G}_{i}$, inequality (12) holds. Thus, there is a higher probability of reaching the target concentration range when Diaz et al.'s algorithm is used instead of formula (10).

Even more, a slight modification of the proof of Theorem 3.1 in Diaz et al. (2007) allows proving that inequality (12) is also valid for $i \geq 2$ when the initial dosage $D_{1}$ is other than that given by Eq. (4), provided that dosage adjustments for patient $A$ are performed using Eqs. (5) and (6) with $i=2, \ldots, n+1$. This should not be a surprise, because, in general, the distribution of $Y_{D_{j}} / D_{j}$ does not depend on the method used to compute $D_{j}$, although, of course, the distribution of $Y_{D_{j}}$ does. 
A further modification of the proof also allows proving that if $\left(D_{i}, Y_{D_{i}}\right), i=$ $1, \ldots, n$ are $n$ steady-state dosage-concentration pairs provided by patient $A$ in other circumstances, for instance, during a TDM that did not necessarily implement Diaz et al.'s algorithm, and if $Y_{D_{i}} / D_{i}, i=1, \ldots, n$, are mutually and conditionally independent given $\gamma$, then inequality (12) is also valid for $i=n+1$, provided that $D_{n+1}$ is computed using Eqs. (5) and (6) with $i=n+1$. Interestingly, under these more general conditions, if $i^{*}$ is given by Eq. (18) in Diaz et al. (2007) and $n+1 \geq i^{*}$, then the probability that $D_{n+1}$ is $\omega$-optimum is at least $p$, that is, $D_{n+1}$ still enjoys an optimality property; however, due to inequality $(12), D_{b, n+1}$ may not have this property. Finally, if $n+1<i^{*}$, then the clinician may use $D_{n+1}$ as an initial dosage for patient $A$ and perform at least $i^{*}-n-1$ algorithm steps in order to obtain a dosage that is $\omega$-optimum for patient $A$ with a probability of at least $p$.

In summary, our computations show that formula (5) may produce better results than formula (10), even if the dosages and concentrations inputted to formula (6) are not obtained through a complete or strict application of Diaz et al.'s algorithm. Moreover, if the number of dosage-concentration pairs is sufficient $\left(\geq i^{*}\right)$, only 1 application of formula (5) may be enough to obtain a desired dosage optimality, regardless of how the pairs were obtained. The objective of a complete application of the algorithm, however, is to ensure that the probability of the drug concentration falling within the target range is maximized each time a dosage is computed.

\section{Application to clozapine individualization}

Clozapine is an antipsychotic drug used to treat patients with severe schizophrenia. Diaz et al. (2008) fit a linear model with only a random intercept and fixed-effect covariates to 415 steady-state (trough) plasma clozapine concentrations provided by 255 schizophrenia patients. Some schizophrenia patients need to be treated not only 
for their psychotic symptoms but also for depression with antidepressants or seizures with anticonvulsants. Thus, the objective of the above study was to measure the size of co-medication effects on plasma clozapine concentrations, using a measure of average effect size that is also described in Botts et al. (2008) and de Leon et al. (2007a, 2007b) and is based on relative percentiles (Muñoz and Xu, 1996; Diaz et al., 2007). The fitted model was similar to model (2), except that $\boldsymbol{\eta}-\boldsymbol{\mu}_{\eta}$ was assumed to be 0; that is, no random effects for the investigated covariates were included. In Diaz et al.'s study (2008), $Y_{D}$ was the steady-state plasma clozapine concentration produced by the clozapine dosage $D$, and the covariates included in the final model were: taking fluoxetine, taking fluvoxamine, taking paroxetine, taking phenobarbital, taking valproic acid, smoking, and the natural log of clozapine dosage $(\log (D))$. All covariates except the latter were dichotomous. Also, an interaction between taking valproic acid and smoking was included in the model.

To examine whether some of the above covariates have random effects, we reanalyzed Diaz et al. (2008) data (Table 1). SAS PROC MIXED was used for computations (Littell et al., 2006). As a result, we found that the model in Diaz et al. (2008) could be improved by including random effects for the covariates, smoking and taking fluoxetine. These random effects were statistically significant at the 0.05 level of significance. Also, Akaike information criteria were 405.3 and 385.6 for the model in Diaz et al. (2008) and the model with covariate random effects, respectively, suggesting that the latter fit better. Table 1 describes the new model. Residual analyses suggested that the model fit well. When the fifth column in Table 1 is compared with the corresponding column in Table 2 of Diaz et al. (2008), we observe that excluding the random effects of smoking and taking fluoxetine from the model did not substantially affect the estimates for the average effect sizes of all covariates included in the model. At the 0.05 level of significance, the statistical significance of these estimates and of fixed-effect estimates 
was not affected either.

By using Eq. (14) in Diaz et al. (2007), $l_{1}=350 \mathrm{ng} / \mathrm{ml}, l_{2}=600 \mathrm{ng} / \mathrm{ml}$, and the error variance $\sigma_{\epsilon}^{2}$ of the model in Table 1, which includes random effects for smoking $\left(Z_{1}\right)$ and taking fluoxetine $\left(Z_{2}\right)$, we obtain $m=0.90$. Moreover, by using Eq.

with $\boldsymbol{Z}=\left(Z_{1}, Z_{2}\right)^{T}$, the values of $\rho(\boldsymbol{Z})$ are calculated as $\rho(1,1)=0.95, \rho(1,0)=0.93$, $\rho(0,1)=0.91$ and $\rho(0,0)=0.88$. Thus, if we use formulae (16) and (18) in Diaz et al. (2007) as explained in Section 2, and assume that the model in Table 1 is used to implement an individualization algorithm in particular patients, we can compute that 3 algorithm steps are sufficient to reach a 0.9 -optimum dosage for at least $95 \%$ of the patients, regardless of the value of $\boldsymbol{Z}$. In other words, to calculate such a dosage for a particular patient, only 2 blood samples would be required from the patient. [Note that $i^{*}$ does not depend on $Z$ in this particular example; however, in general, $i^{*}$ does not always take on the same value for all values of $\boldsymbol{Z}$.]

\section{Computer simulations assuming accurate parameter estimates}

We conducted a simulation study that compared the performance of Diaz et al.'s algorithm with that of an iterative application of formula (10), assuming that the model described in Table 1 is a reasonable representation of the patient population, and using $l_{1}=350 \mathrm{ng} / \mathrm{ml}$ and $l_{2}=600 \mathrm{ng} / \mathrm{ml}$. The main purpose of these simulations was to explore differences between the two approaches, using realistic values for model (2) parameters. The issue of whether and how parameter estimates can be improved is out of the scope of this article and is the topic of a great deal of research (see, e.g., Verbeke and Molenberghs, 2000).

The simulation program was written with SAS/IML [SAS Institute, Inc., 2008]. For each combination of values of the covariates included in the model, a population of 10000 patients that satisfied the model was simulated. Specifically, a simulated 
realization of the random vector $(\psi, \boldsymbol{\eta})$ represented "a patient". In the two approaches, initial dosages were computed with formula (4) (Table 2). For each hypothetical patient, the clozapine dosage was adjusted 3 times using Diaz et al.'s algorithm; that is, 4 steps of the algorithm were implemented. After each algorithm step $i$, the proportion $p_{i}$ of patients whose steady-state plasma clozapine concentration reached the target concentration range, $350-600 \mathrm{ng} / \mathrm{ml}$ was calculated, $i=1, \ldots, 4$. (That is, $p_{i}=P(350<$ $\left.Y_{D_{i}}<600\right) \times 100$.) The "patients" were also used to simulate a traditional TDM in which clozapine dosages were iteratively adjusted 3 times using formula (10) with $C_{0}=C_{0}^{*}$. For this simulated TDM, the proportion of patients whose clozapine concentration reached the target range after the $(i-1)$-th dosage adjustment was computed and denoted by $q_{i}, i=2,3,4$; and the proportion of patients who reached the target range after receiving the initial dosage was denoted by $q_{1}$. Since the initial dosages were the same in both simulated clinical procedures, $p_{1}=q_{1}$. Since oral clozapine dosages are available as multiples of $25 \mathrm{mg}$, dosages computed with formulas (4) and (5), or with formula (10), were rounded to their closest multiple of 25 before "administering" them to the patients (that is, before using the model to compute the resultant plasma clozapine concentrations). All dosages were of at least $25 \mathrm{mg} /$ day.

Table 3 shows the simulation results for non-smokers. Specifically, the table shows the obtained values of $p_{i}$ and $q_{i}, i=1, \ldots, 4$, for each possible combination of covariate values. As seen in Table 3 , for any combination of covariate values, $p_{i}>q_{i}, i=2, \ldots, 4$. For instance, after performing the third dosage adjustment with Diaz et al.'s algorithm in non-smokers who were taking only valproic acid as a comedication, the proportion of patients who reached the target concentration range was $p_{4}=84.4 \%$. In contrast, after the third dosage adjustment with formula (10) in comparable patients, the proportion was lower, $q_{4}=68.6 \%$ (Table 3 ). In general, when compared with the dosage adjustments performed with formula (10), the dosage adjustments performed with Diaz et al.'s 
algorithm always yielded dosages that had a substantially higher likelihood of producing clozapine concentrations within the target range.

Interestingly, $p_{1}<p_{2}<p_{3}<p_{4}$, regardless of the patient subpopulation (Table 3). In contrast, in most subpopulations, $q_{3} \approx q_{4}$. Thus, after 2 dosage adjustments, the traditional TDM did not produce any additional improvement in the dosages. Diaz et al.'s algorithm, however, was able to further improve the dosages in the third dosage adjustment. Conclusions from the simulation results for smokers were essentially the same as those from non-smokers.

In summary, even after accounting for the fact that oral clozapine dosages are available only in discrete amounts that are multiples of $25 \mathrm{mg}$, Diaz et al.'s clinical algorithm produced better dosages than a TDM based on formula (10), in that the algorithm may produce substantially higher proportions of patients reaching the target clozapine concentration range.

\section{A comparison with traditional TDM under parameter estimation errors}

In Section 5, the parameters used to compute dosages with Diaz et al.'s algorithm were the same as those used to simulate the subjects' pharmacokinetic responses to dosages from both the algorithm and formula (10). Thus, it was assumed that the available parameter estimates were very precise estimations of the true model parameters. In practice, parameter estimates must be used by the clinician to compute dosages with Diaz et al.'s algorithm; however, these estimates may not be exactly the same as the true parameters that generate the actual pharmacokinetic responses via model (2). Diaz et al. (2007) explored through simulations the effect of parameter estimation errors on algorithm performance by using a model of plasma clozapine concentrations that was built with data from a double-blind clozapine trial. Although they did not compare the performance of their algorithm with that of other approaches, they found 
that, unless model parameters are very far from their estimates, the algorithm still performs well when the estimates are used in dosage computations in place of the true parameters. Specifically, even when the potentially true parameters were all at a distance of 1 standard error from their estimates, there was a high probability that $D_{i^{*}}$ be 0.9-optimum (Diaz et al., 2007). Considering that maximum likelihood estimators are consistent and usually utilized in the estimation of linear model parameters, Diaz et al.'s simulation results increase our confidence in their algorithm.

In this Section, we use simulations to compare Diaz et al.'s algorithm with formula (10) under the assumption that the form of the linear model representing the patient population is the same as that described in Table 1 , but incorporating the possibility that parameter-estimate values may not be exactly the same as the true parameter values. Observe that the model in Table 1 has 13 real-valued parameters, including variances and all elements of the fixed-effects vector. Following an approach to simulation similar to that in Diaz et al. (2007; p. 2066), let $\vartheta_{i}, i=1, \ldots, 13$, denote the true parameters. Let $\hat{\vartheta}_{i}$ be the estimate of $\vartheta_{i}$ and $\operatorname{se}\left(\hat{\vartheta}_{i}\right)$ its corresponding standard error, $i=1, \ldots, 13$; these are numbers described in Table 1 . For a fixed $r>0$, consider the set $S_{r}$ of 13 -tuples $\left(\vartheta_{1}, \ldots, \vartheta_{13}\right)$ for which the ratio $\left(\hat{\vartheta}_{i}-\vartheta_{i}\right) / \operatorname{se}\left(\hat{\vartheta}_{i}\right)$ is either $r$ or $-r$ for all $i$. That is, $S_{r}$ is the set of all possible combinations of potentially true parameter values that are at a distance of $r$ standard errors from their corresponding estimates. Observe that, for fixed $r, S_{r}$ has $2^{13}=8192$ elements.

As in Section 5, we used a target plasma clozapine concentration range of 350$600 \mathrm{ng} / \mathrm{ml}$; and $p_{3}$ and $q_{3}$ were defined as the proportion of patients who reached this range after 2 dosage adjustments using Diaz et al.'s algorithm and formula (10), respectively. (Recall that $i^{*}$ was estimated to be 3 in Section 4.) For each patient subpopulation determined by the values of $\boldsymbol{X}$ and $\boldsymbol{Z}$, and $r=0.2,0.4, \ldots, 1.6,1.8$, we computed $p_{3}$ and $q_{3}$ for each element of $S_{r}$ by assuming that such element was 
the true combination of parameter values. Each computation of $p_{3}$ and $q_{3}$ used 10000 simulated patients. A particular value of the vector $(\psi, \boldsymbol{\eta})$, which represented a patient, was generated by using the assumed true values of $\mu_{\psi}, \sigma_{\psi}^{2}, \boldsymbol{\mu}_{\eta}$ and $\boldsymbol{V}_{\eta}$. Regardless of the drug individualization method, the pharmacokinetic response of the patient to a dosage $D$ was simulated by first generating one value of the random error $\epsilon$, using the assumed true value of $\sigma_{\epsilon}^{2}$; then, $Y_{D}$ was computed with the formula $D^{d} e^{\alpha+\boldsymbol{\beta}^{T} \boldsymbol{X}+\epsilon}$, using $\alpha=\psi+\boldsymbol{\eta}^{T} \boldsymbol{Z}$ and the assumed true values of $\boldsymbol{\beta}$ and $d$. Independent random errors were used across iterations of Diaz et al.'s algorithm or formula (10), and across the two individualization procedures. Dosages were always rounded to the closest, positive multiple of 25 before computing $Y_{D}$. To compute dosages with Diaz et al.'s algorithm, parameter estimates in Table 1 were entered into formulas (4)-(7). (The parameter estimates were not used to compute dosages with the traditional TDM approach, since formula (10) does not require them.) As in Section 5, the initial dosages used in both individualization procedures were those shown in Table 2 , and we let $C_{0}=C_{0}^{*}$ in formula (10).

For fixed $r$, the number of elements of $S_{r}$ for which $p_{3}>q_{3}$ was computed, divided by $2^{13}$ and multiplied by 100 ; the resultant percentage, denoted by $\Omega_{r}$, was interpreted as the proportion of potentially true parameter combinations in $S_{r}$ under which Diaz et al.'s algorithm outperformed formula (10) after 2 dosage adjustments. Table 4 shows $\Omega_{r}$ for all subpopulations and particular values of $r$. Remarkably, for all patient subpopulations, $\Omega_{r}>98 \%$ when $r \leq 1.2$. (Regardless of the subpopulation investigated, $\Omega_{r}=100 \%$ when $r \leq 1$.) Another computation showed that, when $r \leq 1$, the average of the differences $p_{3}-q_{3}$, taken over all elements of $S_{r}$ was $\geq 8.6 \%$. This suggests that, even if all true parameters are at a distance of 1 standard error from their corresponding estimates in Table 1, a substantially higher percentage of patients may reach the target concentration range if Diaz et al.'s algorithm is used in place of 
the traditional TDM method.

Starting from $r=1.0, \Omega_{r}$ gradually decreased as $r$ increased. For instance, among non-smokers who were taking phenobarbital as their only comedication, $\Omega_{1.0}=100 \%$ $\Omega_{1.2}=99.9 \%, \Omega_{1.4}=88.6 \%, \Omega_{1.6}=78.0 \%$ and $\Omega_{1.8}=75.0 \%$ (Table 4). However, even when $r=1.6$, which is an appreciable distance between an estimate and its corresponding parameter, $\Omega_{r}$ was relatively large in most subpopulations of patients (Table 4). In fact, for 56 out of the 64 subpopulations, $\Omega_{1.6} \geq 80 \%$; for all subpopulations, $\Omega_{1.6} \geq 75 \%$.

We conclude that if the true values of the parameters of the clozapine model reported in Table 1 are not very far from the reported estimates (i.e., $\leq 1.2$ standard errors), Diaz et al.'s algorithm will still be superior in performance to the traditional individualization approach based on formula (10). If moderately higher levels of error affected parameter estimation (between 1.2 and 1.6 standard errors), there is still an acceptable likelihood that the former outperforms the latter approach. These simulation results are consistent with those of Diaz et al. (2007), who found that their algorithm is robust to errors in the estimation of model parameters, at least in the context of clozapine individualization.

\section{Discussion}

This article examines the algorithm proposed by Diaz et al. (2007), which is a rational approach to computing individualized optimum dosages that is based on a precise definition of dosage optimality. Diaz et al. (2007) assumed that the patient belongs to a population that can be described by model (1). This model represents patients' heterogeneity through both covariates and a random intercept. As described in Section 2, the algorithm can be applied to a more general situation, described by model (2), which has 2 additional features: covariates with random effects that add more flexibility 
to heterogeneity modeling, and a regression coefficient $d$ for the log of dosage that may reflect nonlinear pharmacokinetics when different from 1 (Hu and Zhou, 2008; Hu et al., 2009). This suggests that Diaz et al.'s algorithm may have wide applicability.

According to Diaz et al.'s algorithm, once the parameters in model (2) are estimated using population data from phases III or IV, parameter estimates are used to compute a recommended initial dosage $D_{1}$. Then, after a few dosage adjustments, these parameter estimates are combined with measurements from a particular patient in order to compute a dosage that is optimum for the patient with a high probability. Parameter estimates are also used in these dosage adjustments.

There is empirical evidence that model (2) may be a useful tool for describing the effects of covariates on drug steady-state concentrations (Diaz et al., 2007, 2008; Botts et al., 2008; Hu and Zhou, 2008). Some less general versions of this model have been used to compute average dosage correction factors that allow incorporating covariate information in the design of dosage regimes. Our results show that the model may also be used to incorporate individual information that is not explained by available covariates, which is modeled through random regression coefficients. This approach may benefit some patients whose pharmacokinetic or pharmacodynamic characteristics are considerably different from those of an average patient with comparable covariate values.

A version of model (2) that is essentially a random intercept linear model was used by Hu and Zhou (2008) in the analyses of phase III data from 3 different drugs; their data were obtained from very large patient samples. Similar linear models were used by Diaz et al. (2008) and Botts et al. (2008) in analyses of phase IV data. Hu and Zhou (2008) used their linear model only as a method for investigating the robustness of results obtained through compartmental pharmacokinetic models. However, they found a close similarity between covariate-based, average dosage adjustment factors 
computed with compartmental models and those computed with their linear model, which supports the validity of the linear model as a description of their patient samples and investigated drugs. Thus, although $\mathrm{Hu}$ and Zhou did not originally intend to gather evidence in favor of model (2), their work nicely provides very strong empirical and theoretical support for this model. Hu and Zhou appear to be aware of this fact, since they explicitly suggest the possibility that this model may be used to perform primary analyses for regulatory submissions, at least in some situations. (These authors, unfortunately, do not explicitly describe these situations.) This suggestion is also made by $\mathrm{Hu}$ et al. (2009).

A reasonable explanation for the above similarity between results from model (2) and those from compartmental models may be that, in the case of $Y_{D}$ being steadystate drug plasma concentration, model (2) is essentially a model of apparent clearance when the drug follows linear pharmacokinetics, that is, when $d$ is 1 or close to $1(\mathrm{Hu}$ and Zhou, 2008; Hu et al., 2009), and apparent clearance is the main determinant of the association between drug dosage and steady-state plasma concentrations (Winter, 2004). In $\mathrm{Hu}$ et al. (2009), an agreement between the pharmacokinetic conclusions from linear and nonlinear modeling was also observed in a drug that did not follow linear pharmacokinetics; in our opinion, this confirms the potentially wide applicability of linear mixed models to drug dosage computations. In general, linear models are easier to build and fit to phase III or IV data than the nonlinear models that are at the core of traditional pharmacokinetic methods. Thus, model (2) may be a useful tool to compute dosages that are adjusted for covariate values or other individual information. The sparse sampling designs that usually characterize phase III and IV studies, which impede a clear determination of absorption parameters (Jiao et al., 2009), may also be a reason for preferring model (2) when using data from these studies. Sparse sampling is also a reason for focusing only on steady-state concentrations when using phase III 
or IV data (Hu and Zhou, 2008; Jiao et al., 2009). Its use in primary analyses for regulatory submissions is a potential application of model (2), as suggested by Hu and Zhou (2008) and $\mathrm{Hu}$ et al. (2009). More research is needed to explore this possibility and to examine whether the inclusion of covariates with random effects may provide more precise dosage computations.

\section{Conclusion}

The main conclusion of this investigation is that, if model (2) holds, Diaz et al.'s algorithm may produce better personalized dosages than some traditional dosage-adjustment methods used in TDM. This conclusion, along with empirical findings showing that linear mixed models may provide accurate representations of pharmacokinetic data and the fact that linear models are much easier to handle than the nonlinear models that are traditionally used in population pharmacokinetics, suggest a potential use of linear mixed models in drug dosage computation. Moreover, the results in Section 3 show that the algorithm may easily be modified to incorporate steady-state concentrations previously measured through another dosage adjustment procedure, provided that there is no evidence that apparent clearance or covariate values in the patient have substantially changed. [If the values in $X$ have not changed, the stability of apparent clearance may be monitored by examining the stability of the empirical Bayes estimates of $\alpha$, which coincide with the $\widehat{\alpha}_{i}^{\prime}$ 's when the algorithm is applied (Verbeke and Molenberghs, 2000)]. Also, if for some particular reason the clinician decides to change the dosage computed with formula (5) at a particular algorithm step (perhaps because of an observed unwanted reaction from the patient, or just because he/she has to administer a dosage rounded to the closest available dosage), the clinician may still incorporate the obtained concentration into formula (6), treating this concentration as if it were one obtained through an application of the algorithm. Our results show that the dosage computed 
at the next algorithm step will have the same optimality property as the dosage that would be obtained if the clinician had not changed the dosage. Future clinical studies that compare Diaz et al.'s algorithm with dosage-adjustment methods routinely used in TDM are needed in order to quantify the clinical importance of differences between dosages. More research is needed to investigate how to deal with changes in covariate values or apparent clearance during an application of the algorithm; and to explore the implications for the algorithm's performance of not considering, at the populationmodeling stage, variables with important effects on $Y_{D}$.

\section{Acknowledgments}

The clozapine study described in this article was conducted at the Department of Clinical and Experimental Medicine and Pharmacology, University of Messina and IRCCS Centro Neurolesi "Bonino-Pulejo", Messina, Italy. In 1997, Dr. de Leon lectured once supported by Novartis. In the last year (since 11/1/09) he has taken part in a NIH grant in collaboration with Genomas. He has never been a consultant for a pharmaceutical or pharmacogenetic company. Miss Cogollo was partially supported by grant "Apoyo a Estudiantes Sobresalientes de Posgrado" from the Universidad Nacional, Colombia. Dr. Diaz was partially supported by the "Vicedecanatura de Investigación" of the Faculty of Sciences of the Universidad Nacional, Medellin. Dr. Diaz's involvement in this work lasted $4 \frac{1}{2}$ years; the first three years were at the Universidad Nacional, Medellin, Colombia, and the remaining $1 \frac{1}{2}$ years at the University of Kansas, Kansas City, KS. In the last year, Dr. Spina has lectured supported by Eli Lilly, Janssen, AstraZeneca and Bristol-Myers Squibb. Lorraine Maw, M.A., helped edit the article. 


\section{References}

[1] Botts, S., Diaz, F. J., Santoro, V., Spina, E., Muscatello, M. R., Cogollo, M., Castro, F. E., de Leon, J. (2008). Estimating the effects of co-medications on plasma olanzapine concentrations by using a mixed model. Progress in NeuroPsychopharmacology \&6 Biological Psychiatry. 32: 1453-1458.

[2] Cuadrado, M.J., Khamashta, M.A. (2000). The anti-phospholipid antibody syndrome (Hughes syndrome): therapeutic aspects. Bailliere's Clinical Rheumatology. 14: $151-163$.

[3] de Leon, J., Susce, M.T, Johnson, M., Hardin, M., Pointer, L., Ruao, G., Diaz, F.J. (2007a). A clinical study of the association of antipsychotics with hyperlipidemia. Schizophrenia Research. 92: 95-102.

[4] de Leon, J., Susce, M.T., Pan, R.M., Wedlund, P.J., Orrego, M.L., Diaz, F.J. (2007b). A study of genetic (CYP2D6 and ABCB1) and environmental (drug inhibitors and inducers) variables that may influence plasma risperidone levels. Pharmacopsychiatry. 40: 93-102.

[5] Diaz, F.J., Rivera, T.E., Josiassen, R.C., de Leon, J. (2007). Individualizing drug dosage by using a random intercept linear model. Statistics in Medicine. 26: 20522073.

[6] Diaz, F.J., Santoro, V., Spina, E., Cogollo, M., Rivera, T.E., Botts, S., de Leon, J. (2008). Estimating the size of the effects of co-medications on plasma clozapine concentrations using a model that controls for clozapine doses and confounding variables. Pharmacopsychiatry. 41: 81-91.

[7] Dole, V.P. (1980). Addictive behavior. Scientific American. (December) 243(6): 138-154. 
[8] Hu, C., Zhang, J., Zhou, H. (2009). Confirmatory analysis for phase III population pharmacokinetics. Pharmaceutical Statistics. (www.interscience.wiley.com) DOI:10.1002/pst.403.

[9] Hu, C., Zhou, H. (2008). An improved approach for confirmatory phase III population pharmacokinetic analysis. Journal of Clinical Pharmacology. 48: 812-822.

[10] Jiao, Z., Shi, X.J., Li, Z.D., Zhong, M.K. (2009). Population pharmacokinetics of sirolimus in de novo Chinese adult renal transplant patients. British Journal of Clinical Pharmacology. 68: 47-60.

[11] Littell RC, Milliken GA, Stroup WW, Wolfinger RD. SAS for Mixed Models. 2nd ed. SAS Institute, Inc.: Cary, NC, 2006.

[12] Molenberghs, G., Burzykowski, T., Alonso, A., Assam, P., Tilahun, A., Buyse, M. (2008). The meta-analytic framework for the evaluation of surrogate endpoints in clinical trials. Journal of Statistical Planning and Inference. 138: 432-449.

[13] Muñoz, A., Xu, J. (1996). Models for the incubation of AIDS and variations according to age and period. Statistics in Medicine. 15: 2459-2473.

[14] Nies, A.S. (2001). Principles of therapeutics. In: The pharmacological basis of therapeutics, 10th ed. Hardman JG, Limbird LE, Gilman AG (Eds). McGrawHill: New York, NY.

[15] Pillai, G., Mentré, F., Steimer, J-L. (2005). Non-Linear Mixed Effects Modeling From Methodology and Software Development to Driving Implementation in Drug Development Science. Journal of Pharmacokinetics and Pharmacodynamics. 32: 161-183.

[16] Sabaawi, M., Singh, N.N., de Leon, J. (2006). Guidelines for the use of clozapine in individuals with developmental disabilities. Research in Developmental Disabilities. 27: 309-336. 
[17] SAS Institute, Inc. (2008). SAS/IML 9.2 User's Guide. Cary, NC: SAS Institute, Inc.

[18] Sheiner, L.B., Beal, S.L. (1980). Evaluation of methods for estimating population pharmacokinetic parameters. I. Michaelis-Menten model: Routine clinical pharmacokinetic data. Journal of Pharmacokinetics and Biopharmaceutics. 8: 553-571.

[19] Sheiner, L.B., Beal, SL. (1982). Bayesian individualization of pharmacokinetics: Simple implementation and comparison with non-Bayesian methods. Journal of Pharmaceutical Sciences. 71: 1344-1348.

[20] Shirrell, D.J., Gibbar-Clements, T., Dooley, R., and Free, C. (1999). Understanding Therapeutic Drug Monitoring. American Journal of Nursing. 99: 42-44.

[21] Verbeke, G., Molenberghs, G. (2000). Linear Mixed Models for Longitudinal Data. Springer-Verlag: New York.

[22] Vozeh, S., Muir, K.T., Sheiner, L.B., Follath, F. (1981). Predicting individual phenytoin dosage. Journal of Pharmacokinetics and Biopharmaceutics. 9: 131146.

[23] Whiting, B., Kelman, A.W., Grevel, J. (1986). Population pharmacokinetics. Theory and clinical application. Clinical Pharmacokinetics. 11: 387-401.

[24] Winter, M.E. (2004). Basic Clinical Pharmacokinetics. 4th Edition. Lippincott Williams \& Wilkins: Philadelphia, PA. 
Table 1: Fixed effects and effect sizes $(E)$ for variables significantly associated with the natural log of plasma clozapine concentration, according to a linear mixed effects model that includes random effects for smoking and taking fluoxetine $(N=255)^{a}$.

\begin{tabular}{|c|c|c|c|c|c|}
\hline Variable & Fixed effects ${ }^{b}$ & $95 \% \mathrm{CI}^{c, d}$ & P value ${ }^{e}$ & $E^{f}$ & $95 \% \mathrm{CI}^{g}$ \\
\hline Taking fluoxetine ${ }^{h}$ & 0.33 & $(0.15,0.51)$ & 0.0013 & $+39 \%$ & $(16,67)$ \\
\hline Taking fluvoxamine ${ }^{h}$ & 1.25 & $(1.04,1.47)$ & $<0.001$ & $+249 \%$ & $(183,335)$ \\
\hline Taking paroxetine ${ }^{h}$ & 0.26 & $(0.12,0.39)$ & $<0.001$ & $+30 \%$ & $(13,48)$ \\
\hline Taking phenobarbital ${ }^{h}$ & -0.34 & $(-0.53,-0.14)$ & $<0.001$ & $-29 \%$ & $(-41,-13)$ \\
\hline Taking valproic acid ${ }^{h}$ & 0.14 & $(0.014,0.27)$ & 0.03 & & \\
\hline - Non-smokers & & & & $+15 \%$ & $(1,31)$ \\
\hline - Smokers & & & & $-22 \%$ & $(-44,8)$ \\
\hline Smoking $^{i}$ & -0.24 & $(-0.42,-0.055)$ & 0.011 & & \\
\hline - Not taking valproic acid & & & & $-21 \%$ & $(-34,-5)$ \\
\hline - Taking valproic acid & & & & $-47 \%$ & $(-62,-25)$ \\
\hline \multicolumn{6}{|l|}{ Valproic acid-smoking } \\
\hline interaction & -0.39 & $(-0.74,-0.038)$ & 0.03 & & \\
\hline $\log ($ dose $)$ & 1.23 & $(1.09,1.38)$ & $<0.001$ & & \\
\hline
\end{tabular}

95\% CI $=95 \%$ confidence interval. This table looks similar to Table 2 in Diaz et al. (2008) but describes a different model.

a Following the notation in Section 2, the model for plasma clozapine concentrations was $\log (C)=\alpha-0.24 Z_{1}+0.33 Z_{2}+1.25 X_{1}+0.26 X_{2}-0.34 X_{3}+0.14 X_{4}-0.39 X_{5}+1.23 \log (D)+\epsilon$, where $Z_{1}=$ smoking, $Z_{2}=$ taking fluoxetine, $X_{1}=$ taking fluvoxamine, $X_{2}=$ taking paroxetine, $X_{3}=$ taking phenobarbital, $X_{4}=$ taking valproic acid, $X_{5}=Z_{1} X_{4}, C=$ plasma clozapine concentration, and $D=$ clozapine dosage. The intra-individual variation, $\sigma_{\epsilon}^{2}$ was 0.027 [95\% CI, $\left.(0.022,0.035)\right]$. The mean of $\psi$ was $-1.05(-1.89,-0.21)$. If $\boldsymbol{\eta}$ is the vector of random regression coefficients corresponding to $\boldsymbol{Z}=\left(Z_{1}, Z_{2}\right)^{T}$, then the variance-covariance matrix of the random effects, $\operatorname{Var}\left[\left(\psi-\mu_{\psi},\left(\boldsymbol{\eta}-\boldsymbol{\mu}_{\eta}\right)^{T}\right)^{T}\right]$ was a diagonal matrix whose components were as follows: variance of $\psi, 0.20 \quad(0.16,0.26)$; variance of the coefficient of smoking, $0.19(0.10,0.51)$; and variance of the coefficient of taking fluoxetine, $0.083(0.037,0.34)$. 
${ }^{b}$ The numbers in this column are the components of $\boldsymbol{\mu}_{\eta}, \boldsymbol{\beta}$ or $d$.

c $95 \%$ CI for the fixed effect.

$d$ The standard errors of the fixed-effects estimators were 0.43 (intercept), 0.084 (taking fluoxetine), 0.11 (fluvoxamine), 0.068 (paroxetine), 0.099 (phenobarbital), 0.065 (valproic acid), 0.092 (smoking), 0.1770 (valproic acid-smoking interaction), and 0.075 (log of dose). The standard errors of the variance estimators were 0.024 for the variance of $\psi, 0.044$ for the variance of the coefficient of taking fluoxetine, 0.076 for the variance of the coefficient of smoking, and 0.0034 for the intraindividual variation.

$e$ Tests the null hypothesis that the fixed effect is equal to 0 vs. the hypothesis that it is different from 0 .

$f$ The effect size $E$ measures the (adjusted) percentage change in the mean or median (or any other percentile) of plasma clozapine concentrations due to coadministration of the corresponding drug.

g $95 \%$ CI for $E$.

$h$ The dichotomous variable was defined as 1 if the patient was taking the comedication and 0 otherwise.

${ }^{i}$ The dichotomous variable was defined as 1 if the patient was a smoker and 0 otherwise. 
Table 2: Initial clozapine dosages (in $\mathrm{mg} /$ day) used in simulations. These dosages are optimal for an "average patient" from a population that satisfies the model in Table 1 and a target steady-state, plasma clozapine concentration range of 350-600 $\mathrm{ng} / \mathrm{ml}$.

\begin{tabular}{|c|c|c|c|c|c|c|c|}
\hline \multirow[b]{3}{*}{ Smokers } & \multirow[b]{3}{*}{ Fluvoxamine } & \multirow[b]{3}{*}{ Fluoxetine } & \multirow[b]{3}{*}{ Paroxetine } & \multirow{2}{*}{\multicolumn{2}{|c|}{$\frac{\text { Phenobarbital (No) }}{\text { Valproic Acid }}$}} & \multirow{2}{*}{\multicolumn{2}{|c|}{$\frac{\text { Phenobarbital (Yes) }}{\text { Valproic Acid }}$}} \\
\hline & & & & & & & \\
\hline & & & & No & Yes & No & Yes \\
\hline \multirow{8}{*}{ No } & \multirow{4}{*}{ No } & \multirow[b]{2}{*}{ No } & No & 350 & 300 & 450 & 400 \\
\hline & & & Yes & 275 & 225 & 350 & 300 \\
\hline & & \multirow[b]{2}{*}{ Yes } & No & 250 & 225 & 350 & 300 \\
\hline & & & Yes & 200 & 200 & 275 & 250 \\
\hline & \multirow{4}{*}{ Yes } & \multirow[b]{2}{*}{ No } & No & 125 & 100 & 175 & 150 \\
\hline & & & Yes & 100 & 100 & 125 & 125 \\
\hline & & \multirow[b]{2}{*}{ Yes } & No & 100 & 75 & 125 & 100 \\
\hline & & & Yes & 75 & 75 & 100 & 100 \\
\hline \multirow{8}{*}{ Yes } & \multirow{4}{*}{ No } & \multirow[b]{2}{*}{ No } & No & 425 & 500 & 550 & 675 \\
\hline & & & Yes & 325 & 425 & 450 & 550 \\
\hline & & \multirow[b]{2}{*}{ Yes } & No & 325 & 400 & 425 & 525 \\
\hline & & & Yes & 250 & 325 & 350 & 425 \\
\hline & \multirow{4}{*}{ Yes } & \multirow{2}{*}{ No } & No & 150 & 175 & 200 & 250 \\
\hline & & & Yes & 125 & 150 & 150 & 200 \\
\hline & & \multirow{2}{*}{ Yes } & No & 125 & 150 & 150 & 175 \\
\hline & & & Yes & 100 & 125 & 125 & 150 \\
\hline
\end{tabular}


Table 3: Proportions of non-smokers who reached a target clozapine concentration range of 350-600 $n g / m l$ under Diaz et al.'s algorithm $\left(p_{i}\right)$, and under a TDM method based on formula (10) $\left(q_{i}\right)$.

These simulations assume that the patients satisfy the model in Table 1.

\begin{tabular}{|c|c|c|c|c|c|c|c|c|c|c|c|}
\hline \multirow{3}{*}{ FLUVOX } & \multirow{3}{*}{ FLUOX } & \multirow{3}{*}{ PAROX } & \multirow{3}{*}{ Step $i$} & \multicolumn{4}{|c|}{ PHENOB (No) } & \multicolumn{4}{|c|}{ PHENOB (Yes) } \\
\hline & & & & \multicolumn{2}{|c|}{ VALPRO (No) } & \multicolumn{2}{|c|}{ VALPRO (Yes) } & \multicolumn{2}{|c|}{ VALPRO (No) } & \multicolumn{2}{|c|}{ VALPRO (Yes) } \\
\hline & & & & $p_{i}$ & $q_{i}$ & $p_{i}$ & $q_{i}$ & $p_{i}$ & $q_{i}$ & $p_{i}$ & $q_{i}$ \\
\hline \multirow{16}{*}{ No } & \multirow{8}{*}{ No } & \multirow{4}{*}{ No } & 1 & 42.1 & 42.1 & 43.5 & 43.5 & 43.7 & 43.7 & 42.6 & 42.6 \\
\hline & & & 2 & 76.7 & 66.4 & 75.8 & 65.9 & 75.6 & 66.6 & 76.1 & 65.6 \\
\hline & & & 3 & 82.2 & 68.1 & 81.6 & 67.7 & 81.7 & 68.3 & 81.9 & 68.8 \\
\hline & & & 4 & 84.2 & 68.9 & 84.4 & 68.6 & 84.8 & 68.4 & 84.3 & 69.2 \\
\hline & & \multirow{4}{*}{ Yes } & 1 & 43.1 & 43.1 & 42.9 & 42.9 & 42.7 & 42.7 & 42.20 & 42.2 \\
\hline & & & 2 & 76.8 & 65.8 & 76.2 & 65.8 & 77.1 & 66.8 & 76.4 & 66.3 \\
\hline & & & 3 & 82.2 & 68.2 & 81.2 & 69.1 & 82.3 & 68.4 & 81.5 & 68.9 \\
\hline & & & 4 & 83.8 & 69.3 & 84.1 & 69.0 & 84.7 & 68.6 & 84.1 & 69.4 \\
\hline & \multirow{8}{*}{ Yes } & \multirow{4}{*}{ No } & 1 & 36.8 & 36.8 & 37.5 & 37.5 & 37.5 & 37.5 & 37.5 & 37.5 \\
\hline & & & 2 & 75.6 & 64.7 & 75.8 & 64.1 & 76.5 & 63.8 & 76.3 & 65.1 \\
\hline & & & 3 & 81.7 & 68.5 & 80.8 & 68.0 & 82.2 & 68.5 & 81.6 & 68.0 \\
\hline & & & 4 & 84.0 & 68.5 & 84.2 & 68.5 & 85.0 & 68.9 & 84.5 & 68.5 \\
\hline & & \multirow{4}{*}{ Yes } & 1 & 36.3 & 36.3 & 36.3 & 36.3 & 37.2 & 37.2 & 37.1 & 37.1 \\
\hline & & & 2 & 74.7 & 64.4 & 74.8 & 63.9 & 76.2 & 65.3 & 75.3 & 64.0 \\
\hline & & & 3 & 81.0 & 68.1 & 80.9 & 68.1 & 81.3 & 68.5 & 81.2 & 67.3 \\
\hline & & & 4 & 83.6 & 68.3 & 82.8 & 68.2 & 84.3 & 68.4 & 83.5 & 67.9 \\
\hline \multirow{16}{*}{ Yes } & \multirow{8}{*}{ No } & \multirow{4}{*}{ No } & 1 & 43.2 & 43.2 & 40.8 & 40.8 & 41.1 & 41.1 & 42.2 & 42.2 \\
\hline & & & 2 & 74.1 & 64.3 & 72.5 & 63.5 & 76.1 & 64.8 & 74.4 & 64.8 \\
\hline & & & 3 & 78.7 & 65.8 & 78.0 & 67.0 & 80.3 & 67.9 & 79.4 & 66.6 \\
\hline & & & 4 & 80.7 & 66.0 & 80.9 & 67.0 & 83.2 & 68.4 & 82.0 & 68.2 \\
\hline & & \multirow{4}{*}{ Yes } & 1 & 43.2 & 43.2 & 40.8 & 40.8 & 42.6 & 42.6 & 42.4 & 42.4 \\
\hline & & & 2 & 72.0 & 63.9 & 71.8 & 62.5 & 74.0 & 65.2 & 73.7 & 64.8 \\
\hline & & & 3 & 77.4 & 65.3 & 75.6 & 64.1 & 80.2 & 66.9 & 78.9 & 66.8 \\
\hline & & & 4 & 78.5 & 66.2 & 77.3 & 64.4 & 81.3 & 66.3 & 80.9 & 66.9 \\
\hline & \multirow{8}{*}{ Yes } & \multirow{4}{*}{ No } & 1 & 36.8 & 36.8 & 35.2 & 35.2 & 37.3 & 37.3 & 36.4 & 36.4 \\
\hline & & & 2 & 71.0 & 61.4 & 70.0 & 60.2 & 73.5 & 63.0 & 73.0 & 62.7 \\
\hline & & & 3 & 75.6 & 64.4 & 73.9 & 63.7 & 78.9 & 67.0 & 77.0 & 65.7 \\
\hline & & & 4 & 77.8 & 64.5 & 75.7 & 63.8 & 80.5 & 67.8 & 79.4 & 65.4 \\
\hline & & \multirow{4}{*}{ Yes } & 1 & 37.5 & 37.5 & \begin{tabular}{|l|}
36.2 \\
\end{tabular} & 36.2 & 37.2 & 37.2 & \begin{tabular}{|l|}
36.3 \\
\end{tabular} & 36.3 \\
\hline & & & 2 & 69.0 & 59.1 & 68.1 & 59.0 & 72.1 & 61.9 & 70.7 & 61.1 \\
\hline & & & 3 & 73.4 & 62.5 & 71.4 & 61.5 & 76.7 & 64.3 & 75.4 & 64.3 \\
\hline & & & 4 & 75.3 & 62.5 & 73.7 & 61.5 & 78.5 & 64.8 & 77.1 & 64.5 \\
\hline
\end{tabular}

FLUVOX: fluvoxamine; FLUOX: fluoxetine; PAROX: paroxetine; PHENOB: phenobarbital;VALPRO: valproic acid. 
Table 4: Proportion $\Omega_{r}$ of combinations of potentially true parameters that are at a distance of $r$ standard errors from their estimates under which Diaz et al.'s algorithm outperformed formula (10) after 2 clozapine dosage adjustments, for particular values of $r$ and subpopulations of patients.

\begin{tabular}{|c|c|c|c|c|c|c|c|c|c|c|c|}
\hline \multirow{3}{*}{ FLUVOX } & \multirow{3}{*}{ FLUOX } & \multirow{3}{*}{ PAROX } & \multirow{3}{*}{$r$} & \multicolumn{4}{|c|}{ PHENOB (No) } & \multicolumn{4}{|c|}{ PHENOB (Yes) } \\
\hline & & & & \multicolumn{2}{|c|}{ VALPRO (No) } & \multicolumn{2}{|c|}{ VALPRO (Yes) } & \multicolumn{2}{|c|}{ VALPRO (No) } & \multicolumn{2}{|c|}{ VALPRO (Yes) } \\
\hline & & & & $N S M O$ & $S M O$ & NSMO & $S M O$ & $N S M O$ & $S M O$ & $N S M O$ & $S M O$ \\
\hline \multirow{16}{*}{ No } & \multirow{8}{*}{ No } & \multirow{4}{*}{ No } & $\leq 1.2$ & 100 & 100 & 100 & 100 & $\geq 99.9$ & 100 & $\geq 99.2$ & 100 \\
\hline & & & 1.4 & 88.3 & 100 & 91.3 & 97.4 & 88.6 & 98.8 & 87.8 & 96.5 \\
\hline & & & 1.6 & 75.0 & 92.8 & 77.0 & 88.3 & 78.0 & 88.0 & 78.1 & 88.5 \\
\hline & & & 1.8 & 75.0 & 80.4 & 75.0 & 81.5 & 75.0 & 78.5 & 75.0 & 81.6 \\
\hline & & \multirow{4}{*}{ Yes } & $\leq 1.2$ & 100 & 100 & 100 & 100 & $\geq 99.5$ & 100 & $\geq 98.7$ & 100 \\
\hline & & & 1.4 & 92.3 & 99.8 & 90.2 & 97.9 & 88.4 & 98.8 & 89.5 & 96.8 \\
\hline & & & 1.6 & 78.2 & 90.3 & 78.3 & 90.3 & 78.3 & 90.8 & 79.8 & 88.6 \\
\hline & & & 1.8 & 75.0 & 79.2 & 75.0 & 83.5 & 75.0 & 81.4 & 76.1 & 82.1 \\
\hline & \multirow{8}{*}{ Yes } & \multirow{4}{*}{ No } & $\leq 1.2$ & 100 & 100 & 100 & 100 & 100 & 100 & 100 & 100 \\
\hline & & & 1.4 & 98.1 & 100 & 97.4 & 99.0 & 96.8 & 99.7 & 96.3 & 98.2 \\
\hline & & & 1.6 & 83.3 & 95.5 & 87.1 & 91.7 & 86.9 & 92.5 & 84.7 & 91.2 \\
\hline & & & 1.8 & 76.0 & 84.1 & 77.8 & 84.3 & 78.0 & 82.8 & 77.6 & 83.5 \\
\hline & & \multirow{4}{*}{ Yes } & $\leq 1.2$ & 100 & 100 & 100 & 100 & 100 & 100 & 100 & 100 \\
\hline & & & 1.4 & 97.3 & 100 & 98.5 & 98.9 & 97.0 & 99.6 & 96.5 & 98.5 \\
\hline & & & 1.6 & 86.8 & 93.7 & 90.0 & 92.6 & 85.2 & 94.0 & 87.1 & 91.4 \\
\hline & & & 1.8 & 77.9 & 83.0 & 80.0 & 85.3 & 78.0 & 84.5 & 79.5 & 84.4 \\
\hline \multirow{16}{*}{ Yes } & \multirow{8}{*}{ No } & \multirow{4}{*}{ No } & $\leq 1.2$ & 100 & 100 & 100 & 100 & 100 & 100 & $\geq 99.0$ & 100 \\
\hline & & & 1.4 & 93.6 & 99.9 & 91.2 & 97.8 & 92.9 & 99.1 & 92.0 & 97.7 \\
\hline & & & 1.6 & 81.4 & 94.4 & 81.4 & 90.7 & 83.6 & 92.2 & 83.1 & 91.1 \\
\hline & & & 1.8 & 75.4 & 84.9 & 76.7 & 84.0 & 78.1 & 83.0 & 77.5 & 84.4 \\
\hline & & \multirow{4}{*}{ Yes } & $\leq 1.2$ & 100 & 100 & 100 & 100 & $\geq 98.8$ & 100 & $\geq 99.2$ & 100 \\
\hline & & & 1.4 & 93.6 & 99.8 & 94.7 & 98.3 & 91.7 & 98.9 & 93.2 & 97.9 \\
\hline & & & 1.6 & 84.1 & 93.9 & 85.9 & 92.0 & 82.6 & 91.5 & 84.5 & 91.1 \\
\hline & & & 1.8 & 77.9 & 84.8 & 79.7 & 85.3 & 76.9 & 83.2 & 79.0 & 84.7 \\
\hline & \multirow{8}{*}{ Yes } & \multirow{4}{*}{ No } & $\leq 1.2$ & 100 & 100 & 100 & 100 & 100 & 100 & 100 & 100 \\
\hline & & & 1.4 & 98.9 & 100 & 97.6 & 99.4 & 98.1 & 99.6 & 96.2 & 98.3 \\
\hline & & & 1.6 & 90.1 & 97.2 & 88.5 & 94.2 & 90.4 & 95.0 & 87.5 & 92.1 \\
\hline & & & 1.8 & 82.4 & 89.0 & 80.8 & 87.4 & 82.2 & 86.6 & 80.2 & 85.1 \\
\hline & & \multirow{4}{*}{ Yes } & $\leq 1.2$ & 100 & 100 & 100 & 100 & 100 & 100 & 100 & 100 \\
\hline & & & 1.4 & 98.3 & 100 & 99.0 & 99.3 & 97.5 & 99.6 & 98.1 & 98.7 \\
\hline & & & 1.6 & 90.8 & 96.6 & 92.2 & 95.0 & 88.9 & 95.2 & 91.3 & 93.0 \\
\hline & & & 1.8 & 82.2 & 88.6 & 84.3 & 88.4 & 81.6 & 87.2 & 84.0 & 86.6 \\
\hline
\end{tabular}

FLUVOX: fluvoxamine; FLUOX: fluoxetine; PAROX: paroxetine; PHENOB: phenobarbital;VALPRO: valproic acid; NSMO: non-smoker; SMO:smoker. 